\title{
Efficient mining of pan-correlation patterns from time course data
}

\author{
Qian $\mathrm{Liu}^{1}$, Jinyan $\mathrm{Li}^{1}$, Limsoon Wong ${ }^{2}$, and Kotagiri Ramamohanarao ${ }^{3}$ \\ 1 Advanced Analytics Institute, University of Technology Sydney, 81 Broadway, \\ Ultimo NSW 2007, Australia \\ 2 School of Computing, National University of Singapore, 3 Computing Drive, \\ Singapore 117417 \\ 3 Dept of Computing and Information Systems, the University of Melbourne, \\ Parkville, Victoria 3010, Australia
}

\begin{abstract}
There are different types of correlation patterns between the variables of a time course data set, such as positive correlations, negative correlations, time-lagged correlations, and those correlations containing small interrupted gaps. Usually, these correlations are maintained only on a subset of time points rather than on the whole span of the time points which are traditionally required for correlation definition. As these types of patterns underline different trends of data movement, mining all of them is an important step to gain a broad insight into the dependencies of the variables. In this work, we prove that these diverse types of correlation patterns can be all represented by a generalized form of positive correlation patterns. We also prove a correspondence between positive correlation patterns and sequential patterns. We then present an efficient single-scan algorithm for mining all of these types of correlations. This "pan-correlation" mining algorithm is evaluated on synthetic time course data sets, as well as on yeast cell cycle gene expression data sets. The results indicate that: (i) our mining algorithm has linear time increment in terms of increasing number of variables; (ii) negative correlation patterns are abundant in real-world data sets; and (iii) correlation patterns with time lags and gaps are also abundant. Existing methods have only discovered incomplete forms of many of these patterns, and have missed some important patterns completely.
\end{abstract}

\section{Introduction}

Time course data have been involved in many real-world applications, especially in the fields of finance, healthcare and biomedicine. This work investigates the mining algorithm of correlation patterns. A correlation pattern is defined as a series of highly correlated data movement trends between two sets of variables on some subset of time points (not necessarily on the whole span of the time points). The two basic types of correlations are the positive correlation or the negative correlation. A pattern of positive correlation is a set of variables showing the same direction in their data movements. On the other hand, the data changes of one set of variables in a negative correlation pattern go jointly up or down whenever the value changes of the other set of variables move in the opposite direction. Variables in time course data also have time-dependent interactions. 
The influence of a variable on other variables sometimes may not be immediate. Instead, it is going to be effective only after some time delay, leading to timelagged correlation (positive or negative). Thus, there are four types of correlation patterns: the basic positive and negative correlation patterns (i.e., synchronized correlations without time delay), and time-lagged positive and negative correlation patterns. Time course data in real-world applications may also contain a small amount of unknown noise and errors. These noise and errors can interrupt the time continuity of a correlation, leading to gaps in the correlation. Gaps complicate the complexity of correlation mining, because the gaps can happen at any time point, and the length of a gap is unknown.

This work introduces a new type of correlation pattern, named "pan-correlation patterns", to maximize the sequence of coherent data movements in one pattern. A pan-correlation pattern consists of a maximized sub-list $V_{0}$ of variables, where all the listed variables are associated with a segment of time points having the same length, such that $V_{0}$ can be divided into two not necessarily mutuallyexclusive lists of variables $V_{1}$ and $V_{2}$, satisfying: (i) every pair of variables within $V_{1}$ are positively correlated, or time-lag positively correlated, or time-lag positively correlated with gaps; (ii) every pair of variables within $V_{2}$ are positively correlated, or time-lag positively correlated, or time-lag positively correlated with gaps; and (iii) every pair of variables between $V_{1}$ and $V_{2}$ are negatively correlated, or time-lag negatively correlated, or time-lag negatively correlated with gaps. $V_{1}$ or $V_{2}$ can be empty - in this case, a pan-correlation pattern is simplified as a positive pan-correlation pattern. By our definition, a pan-correlation pattern can cover all of the following characteristics: the basic positively/negatively correlated data movement trends, time-lag effects, and noise/error gaps. However, mining significant pan-correlation patterns is a problem of high complexity. Existing methods are not capable of conducting the mining of pan-correlation patterns. They may only be able to detect a special subtype of pan-correlation patterns, for example, positive correlation patterns by $[6,3]$, or negative correlation patterns by $[14,7]$, or both positive and negative correlations by $[15,4]$, or time-lagged positive correlation patterns by $[5,2]$.

Our work introduces an efficient algorithm for mining significant pan-correlation patterns. We proposed three critical ideas. First, we prove that all the different types of correlation patterns can be represented by a generalized form of positive correlation patterns - viz. pan-correlation patterns. Based on this theory, we can focus on the mining of all positive correlations. Second, the time course data set is transformed into a sequential data set containing sequences of "up", "down", and "no-change", which are the three movement trends of variables. With this data discretization idea $[11,7,9]$, the pan-correlation mining problem can be converted into a sequential pattern mining problem. Central to how we enable the representation of the different types of negative correlation patterns through the generalized form of positive correlation patterns is that we make an opposite-mirror copy [8] of the original sequential data set and then add it to the original data. A cost of adding the mirror copy of the sequential data is that many redundant patterns are produced. Thus, our third new idea is to modify 
a sequential pattern mining algorithm to efficiently prune redundant patterns in the mining process. Our pan-correlation mining algorithm is tested on synthetic time course data sets and four microarray gene expression time course data sets. The synthetic data sets are used to demonstrate the efficiency of our algorithm. The experiments on the gene expression data show that negative correlation patterns are indeed abundant in real-world data sets, and that patterns with different time delays and gaps are common. It is worth noting that pancorrelation patterns are not a kind of pairwise correlation patterns, and it is of high time complexity, if not impossible, to use traditional algorithms, clustering or pairwise correlation, to mine pan-correlation patterns.

The rest of the paper is organized as follows. We define the six types of correlation patterns and their closure property in Section 2. We then describe our pan-correlation mining algorithm in Section 3. After that, we present result$\mathrm{s}$ of our pan-correlation mining algorithm on synthetic and also real-life gene expression time course data sets in Section 4.

\section{Problem formulation}

Let $V$ be a set of $N_{V}$ variables $v_{1}, v_{2}, \ldots, v_{N_{V}}$. Let $T$ be a set of $N_{T}$ consecutive time points $t_{1}, t_{2}, \ldots, t_{N_{T}}$. Here, $t_{j}$ and $t_{j+1}$ in $N_{T}$ are two ordered consecutive time points with $t_{j} \prec t_{j+1}$, indicating that $t_{j}$ precedes $t_{j+1}$. Let $m_{i, j}$ denote the value of variable $v_{i}$ at time point $t_{j}$. A time course data set is then defined by the data matrix $M=\left[m_{i, j}\right]_{N_{V} \times N_{T}}$.

\subsection{Correlation patterns: Definitions}

Definition 1. A positive correlation pattern $p$ is a pair comprising a subset $V_{0}$ of variables in $V$ and a continuous segment $T_{p}$ of time points in $T$ such that, for every pair of consecutive time points from $t_{j}$ to $t_{j+1}$ in $T_{p}$, the values of all variables in $V_{0}$ decrease or increase simultaneously. A positive correlation $p$ is written as $p=\left\langle V_{0}, T_{p}\right\rangle$.

It is possible that the magnitude of the 'decrease' or 'increase' is very small. These slight decrease or increase movements are both considered as 'no-change'. Therefore, these time segments will be considered to have the same value movement in a positive correlation when the variables change their movements very slightly. More formally, for each $v_{i}$, we say the value of $v_{i}$ increases (decreases) from consecutive time point $t_{j}$ to $t_{j+1}$ if it changes by at least $\delta_{i}$, where $\delta_{i}$ is some specified threshold. Under this assumption, a pattern containing only 'no-change' provides less information for high correlation. Therefore, we require that a correlation pattern must contain at least one significant decrease/increase movements. This convention is applied on all definitions, lemmas, and propositions in this work. Please note that $\delta \mathrm{s}$ might also result in information lost in correlation pattern. Optimal $\delta$ 's threshold should consider the tradeoff between insignificant, noise change and information lost. There is no gold standard to provide the best $\delta \mathrm{s}$. Thus, the threshold of $\delta \mathrm{s}$ could be specified by users based on domain knowledge.

Definition 2 (Cf. [7]). A negative correlation pattern $n$ is a triplet comprising two non-overlapping subsets $V_{1}$ and $V_{2}$ of variables in $V$ and a continuous segment $T_{n}$ of time points in $T$ such that, for every pair of consecutive time points 
from $t_{j}$ to $t_{j+1}$ in $T_{n}$, the values of all variables in $V_{1}$ decrease while the values of all variables in $V_{2}$ increase, and vice versa. A negative correlation $n$ is written as $n=\left\langle\left(V_{1}, V_{2}\right), T_{n}\right\rangle$.

These two definitions describe a synchronized pace of value change without time delay. In fact, some variables in the data matrix $M$ may have influence on others, but the effect may not take place immediately (i.e., after some time delay).

Definition 3. A time-lagged negative correlation pattern $k n$ is a pair of distinct lists $\left\{\left(v_{x_{1}}, T_{p}^{1}\right), \ldots,\left(v_{x_{h}}, T_{p}^{h}\right)\right\}$ and $\left\{\left(v_{y_{1}}, T_{q}^{1}\right), \ldots,\left(v_{y_{g}}, T_{q}^{g}\right)\right\}$, such that: (i) $V_{1}=\left\{v_{x_{1}}, \ldots, v_{x_{h}}\right\}$ and $V_{2}=\left\{v_{y_{1}}, \ldots, v_{y_{g}}\right\}$ are two possibly overlapping lists of not necessarily distinct variables of $V$; (ii) $T_{K}^{1}=\left\{T_{p}^{1}, \ldots, T_{p}^{h}\right\}$ and $T_{K}^{2}=\left\{T_{q}^{1}, \ldots, T_{q}^{g}\right\}$ are two lists of $h$ and $g$ continuous time segments of the same length in $T$; (iii) for every $1 \leq r<\left|T_{p}^{1}\right|$ and for every $v_{x_{i}} \in V_{1}$, the value of $v_{x_{i}}$ increases (decreases) from the rth time point in $T_{p}^{i}$ to the $(r+1)$ th time point in $T_{p}^{i}$ if and only if for all other $v_{x_{j}} \in V_{1}$, the value of $v_{x_{j}}$ increases (decreases) from the rth time point in $T_{p}^{j}$ to the $(r+1)$ th time point in $T_{p}^{j}$; (iv) for every $1 \leq r<\left|T_{p}^{1}\right|$ and for every $v_{y_{i}} \in V_{2}$, the value of $v_{y_{i}}$ increases (decreases) from the rth time point in $T_{q}^{i}$ to the $(r+1)$ th time point in $T_{q}^{i}$ if and only if for all other $v_{y_{j}} \in V_{2}$, the value of $v_{y_{j}}$ increases (decreases) from the rth time point in $T_{q}^{j}$ to the $(r+1)$ th time point in $T_{q}^{j}$; and $(v)$ for every $1 \leq r<\left|T_{p}^{1}\right|$, for every $v_{x_{i}} \in V_{1}$, and for every $v_{y_{j}} \in V_{2}$, the value of $v_{x_{i}}$ increases (decreases) from the rth time point in $T_{p}^{i}$ to the $(r+1)$ th time point in $T_{p}^{i}$ if and only if the value of $v_{y_{j}}$ decreases (increases) from the rth time point in $T_{q}^{j}$ to the $(r+1)$ th time point in $T_{q}^{j}$. For convenience, a time-lagged negative correlation $k n$ is written as $k n=\left\langle\left(V_{1}, V_{2}\right),\left(T_{K}^{1}, T_{K}^{2}\right)\right\rangle$.

When $V_{1}$ and $T_{K}^{1}$, or $V_{2}$ and $T_{K}^{2}$, are empty, $k n$ is a time-lagged positive correlation, denoted by $k p$.

A time segment can be extended into a discontinuous time segment to tolerate some small amount of noise. For example, $T_{p}=[1,2,3,4,7,8,9,10]$ is a discontinuous time segment containing a gap of length 2 between 4 and 7 . The first 4 time points of $T_{p}$ are continuous from 1 to 4 , and the next 4 time points are continuous from 7 to 10 . The pattern $p=\left\{\left(v, T_{p}=[1,2,3,4,7,8,9,10]\right),\left(v^{\prime}, T_{p}^{\prime}=\right.\right.$ $[1,2,3,4,5,6,7])\}$ is defined as a positive correlation pattern with gaps if the changes of the values of $v$ for any two consecutive time points of $[1,2,3,4]$ are in the same direction as the changes of the values of $v^{\prime}$ for $[1,2,3,4]$, and the changes of the values of $v$ for any two consecutive time points of $[7,8,9,10]$ are in the same direction as the changes of the values of $v^{\prime}$ for $[4,5,6,7]$. The data movement trends between the time points 4 and 7 in $v$ are not considered due to the gap.

Next, we introduce the definitions for (time-lagged) positive/negative correlation patterns that contain gaps. A pair of consecutive time points $t_{i}$ and $t_{i+1}$ is denoted as $\operatorname{tpp}_{(i, i+1)}$. In this work, all time-point pairs are pairs of consecutive time points. Let $T p p=\left\{\operatorname{tpp}_{\left(i_{j}, i_{j}+1\right)} \mid j=1,2, \ldots, h\right\}$ be an ordered list of $h$ time-point pairs, where $t_{i_{j}} \prec t_{i_{j+1}}$. Tpp is continuous if and only if for every $1 \leq k \leq h, i_{k}+1=i_{k+1}$. Otherwise, Tpp is discontinuous and contains gaps. A continuous Tpp corresponds to a continuous time segment. For example, 
$\left\{t p p_{(1,2)}, t p p_{(2,3)}, t p p_{(3,4)}\right\}$ corresponds to time segment $\left\{t_{1}, t_{2}, t_{3}, t_{4}\right\}$. A discontinuous Tpp may also corresponds to a continuous time segment. For example, $\left\{t p p_{(1,2)}, t p p_{(3,4)}\right\}$ correspond to time segment $\left\{t_{1}, t_{2}, t_{3}, t_{4}\right\}$. So, a time segment alone is not sufficient to define the data movements on the time-point pairs and the movement gaps.

Definition 4. A negative pan-correlation pattern is a time-lagged negative correlation pattern with gaps. That is, it is a pair of distinct lists $\left\{\left(v_{x_{1}}, T p p_{p}^{1}\right), \ldots\right.$, $\left.\left(v_{x_{h}}, T p p_{p}^{h}\right)\right\}$ and $\left\{\left(v_{y_{1}}, T p p_{q}^{1}\right), \ldots,\left(v_{y_{g}}, T p p_{q}^{g}\right)\right\}$, such that: (i) $\mathcal{V}_{1}=\left\{v_{x_{1}}, \ldots, v_{x_{h}}\right\}$ and $\mathcal{V}_{2}=\left\{v_{y_{1}}, \ldots, v_{y_{g}}\right\}$ are two possibly overlapping lists of not necessarily distinct variables in $V$; (ii) $\mathcal{T} \mathcal{P} \mathcal{P}_{K}^{1}=\left\{T p p_{p}^{1}, \ldots, T p p_{p}^{h}\right\}$ and $\mathcal{T P} \mathcal{P}_{K}^{2}=\left\{T p p_{q}^{1}, \ldots\right.$, $\left.T p p_{q}^{g}\right\}$ are two lists of time-point-pair lists all with the same length and possibly containing different gaps; (iii) for every $1 \leq r<\left|T p p_{p}^{1}\right|$ and for every $v_{x_{i}} \in \mathcal{V}_{1}$, the value of $v_{x_{i}}$ increases (decreases) at the rth time-point pair in Tpp $p_{p}^{i}$ if and only if for all other $v_{x_{j}} \in \mathcal{V}_{1}$, the value of $v_{x_{j}}$ increases (decreases) at the rth time-point pair in Tpp $p_{p}^{j}$ (iv) for every $1 \leq r<\left|T p p_{p}^{1}\right|$ and for every $v_{y_{i}} \in \mathcal{V}_{2}$, the value of $v_{y_{i}}$ increases (decreases) at the rth time-point pair in Tpp $p_{q}^{i}$ if and only if for all other $v_{y_{j}} \in \mathcal{V}_{2}$, the value of $v_{y_{j}}$ increases (decreases) at the rth time-point pair in Tpp $p_{q}^{j}$; v) for every $1 \leq r<\left|T p p_{p}^{1}\right|$, for every $v_{x_{i}} \in \mathcal{V}_{1}$, and for every $v_{y_{j}} \in \mathcal{V}_{2}$, the value of $v_{x_{i}}$ increases (decreases) at the rth time-point pair in Tpp if and only if the value of $v_{y_{j}}$ decreases (increases) at the rth time-point pair in Tpp $p_{q}^{j}$. For convenience, a negative pan-correlation pattern $\mathcal{C}$ is written as $\mathcal{C}=\left\langle\left(\mathcal{V}_{1}, \mathcal{V}_{2}\right),\left(\mathcal{T} \mathcal{P} \mathcal{P}_{K}^{1}, \mathcal{T} \mathcal{P} \mathcal{P}_{K}^{2}\right)\right\rangle$

When $\mathcal{V}_{1}$ and $\mathcal{T} \mathcal{P} \mathcal{P}_{K}^{1}$, or $\mathcal{V}_{2}$ and $\mathcal{T} \mathcal{P} \mathcal{P}_{K}^{2}$, are empty, $\mathcal{C}$ is a positive pancorrelation. Moreover, every continuous time segment $T *$ in the definitions from Definition 1 to Definition 3 can be converted into a continuous Tpp. Thus all correlation patterns by these definitions can be rewritten by using time-pointpair list $T p p$ to replace time segment $T *$.

There are a huge number of positive and negative pan-correlation patterns in the data matrix $M$. However, we are only interested in those patterns that are closed. A pattern $\mathcal{C}=\left\langle\left(\mathcal{V}_{1}, \mathcal{V}_{2}\right),\left(\mathcal{T} \mathcal{P} \mathcal{P}_{K}^{1}, \mathcal{T} \mathcal{P} \mathcal{P}_{K}^{2}\right)\right\rangle$ is closed if (i) any time-pointpair list in $\mathcal{T P P}_{K}^{1}$ and $\mathcal{T} \mathcal{P} \mathcal{P}_{K}^{2}$ cannot be enlarged to include more time-point pairs without breaking the underlying correlation among the variables in $\mathcal{V}_{1}$ and $\mathcal{V}_{2}$, and (ii) the list of variables $\mathcal{V}_{1}$ and $\mathcal{V}_{2}$ cannot be enlarged to include more variables as there can be no other variable that correlates positively or negatively to those in $\mathcal{V}_{1}$ and $\mathcal{V}_{2}$ over the same number of time-point pairs in $\mathcal{T P} \mathcal{P}_{K}^{1}$ and $\mathcal{T P} \mathcal{P}_{K}^{2}$. Every positive (negative) pan-correlation pattern can be derived from some closed positive (negative) pan-correlation patterns by deleting variables and/or deleting time-point pairs. Thus, the set of all closed positive (negative) pan-correlation patterns forms a lossless and non-redundant representation of positive (negative) pan-correlation patterns. These patterns are called closed patterns and more specifically $\mathbb{C}$-, $\mathbb{C P}$-, and $\mathbb{C N}$-closed patterns.

The following relationships between the various types of pan-correlation patterns can be easily proved. 
Proposition 1. Let $\mathcal{C}=\left\langle\left(\mathcal{V}_{1}, \mathcal{V}_{2}\right),\left(\mathcal{T P} \mathcal{P}_{K}^{1}, \mathcal{T P} \mathcal{P}_{K}^{2}\right)\right\rangle, \mathcal{C}_{1}=\left\langle\mathcal{V}_{1}, \mathcal{T P} \mathcal{P}_{K}^{1}\right\rangle$, and $\mathcal{C}_{2}=\left\langle\mathcal{V}_{2}, \mathcal{T} \mathcal{P} \mathcal{P}_{K}^{2}\right\rangle$. Then

- $\mathcal{C}$ is in $\mathbb{C N}$ implies both $\mathcal{C}_{1}$ and $\mathcal{C}_{2}$ are in $\mathbb{C P}$.

- $\mathcal{C}$ is in $\mathbb{C N}$ if, and only if, $\mathcal{C}^{\prime}=\left\langle\left(\mathcal{V}_{2}, \mathcal{V}_{1}\right),\left(\mathcal{T P} \mathcal{P}_{K}^{2}, \mathcal{T P} \mathcal{P}_{K}^{1}\right)\right\rangle$ is in $\mathbb{C N}$.

$-\mathcal{C}$ is closed in $\mathbb{C}$ if, and only if, it is closed in $\mathbb{C N}$.

- $\mathcal{C}_{1}^{\prime}=\left\langle\left(\mathcal{V}_{1},\{\}\right),\left(\mathcal{T} \mathcal{P} \mathcal{P}_{K}^{1},\{\}\right)\right\rangle$ is closed in $\mathbb{C N}$ implies $\mathcal{C}_{1}$ is closed in $\mathbb{C P}$.

$-\mathcal{C}$ is closed in $\mathbb{C}$ implies for $i \in\{1,2\}$, for every (closed) pattern $\mathcal{C}^{\prime}=$ $\left\langle\mathcal{V}^{\prime}, \mathcal{T} \mathcal{P} \mathcal{P}^{\prime}\right\rangle$ in $\mathbb{C P}$ where $\mathcal{C}_{i} \sqsubseteq_{p} \mathcal{C}^{\prime}$, it is the case that $\mathcal{V}_{i}=\mathcal{V}^{\prime}$ (Note that $\mathcal{C}_{i}=\mathcal{C}^{\prime}$ does not hold).

The second point of Proposition 1 implies some degree of redundancy, as the two patterns $\mathcal{C}$ and $\mathcal{C}^{\prime}$ capture the same correlation information. We will deal with this redundancy later in Section 3.

\subsection{Unified representation of all correlation patterns}

Let $V *$ be a set of variables $v_{1} *, v_{2} *, \ldots, v_{N_{V}} *$. Let $m_{i, j} *=-m_{i, j}$ denote the value of variable $v_{i} *$ at time point $t_{j}$, and this value of $v_{i} *$ at time point $t_{j}$ is the negation of the value of $v_{i}$ at time point $t_{j}$. A negated time course data set is then defined by the data matrix $M *=\left[m_{i, j} *\right]_{N_{V} \times N_{T}}$. It is also called a mirror-copy of $M$. Clearly, whenever the value of $v_{i}$ increases (decreases) from time point $t_{j}$ to time point $t_{j+1}$, the value of $v_{i} *$ decreases (increases) from time point $t_{j}$ to time point $t_{j+1}$. I.e., the value of $v_{i} *$ moves in the opposite direction of $v_{i}$. Let $M^{\prime}$ be the matrix obtained by adding the negated data matrix $M *$ to the original data matrix $M$ (details are given in Section 3.2). Then, the lemma below follows from this observation and can be easily proved.

Lemma 1. $\mathcal{C}=\left\langle\left(\mathcal{V}_{1}=\left\{v_{x_{1}}, \ldots, v_{x_{h}}\right\}, \mathcal{V}_{2}=\left\{v_{y_{1}}, \ldots, v_{y_{g}}\right\}\right),\left(\mathcal{T} \mathcal{P P}_{K}^{1}=\left\{T p p_{p}^{1}, \ldots, T p p_{p}^{h}\right\}\right.\right.$, $\left.\left.\mathcal{T P} \mathcal{P}_{K}^{2}=\left\{T p p_{q}^{1}, \ldots, T p p_{q}^{g}\right\}\right)\right\rangle$ is in $\mathbb{C N}$ in the data matrix $M$ if, and only if, $\mathcal{C} *=$ $\left\langle\mathcal{V}=\left\{v_{x_{1}}, \ldots, v_{x_{h}}, v_{y_{1}} *, \ldots, v_{y_{g}} *\right\}, \mathcal{T P \mathcal { P }}=\left\{T p p_{p}^{1}, \ldots, T p p_{p}^{h}, T p p_{q}^{1}, \ldots, T p p_{q}^{g}\right\}\right\rangle$ is in $\mathbb{C P}$ in the data matrix $M^{\prime}$.

Based on the equivalence above, for $\mathcal{C}$ in $\mathbb{C N}$ with regard to $M$, we write $\mathcal{C} *$ for its counterpart in $\mathbb{C P}$ with regard to $M^{\prime}$.

Every closed $\mathbb{C P}$ pattern in the data matrix $M^{\prime}$ is in a one-to-one correspondence with a closed $\mathbb{C N}$ pattern (also a closed $\mathbb{C}$ pattern) in the data matrix $M$.

Theorem 1. $\mathcal{C}=\left\langle\left(\mathcal{V}_{1}=\left\{v_{x_{1}}, \ldots, v_{x_{h}}\right\}, \mathcal{V}_{2}=\left\{v_{y_{1}}, \ldots, v_{y_{g}}\right\}\right),\left(\mathcal{T P P}_{K}^{1}=\right.\right.$ $\left.\left.\left\{T p p_{p}^{1}, \ldots, T p p_{p}^{h}\right\}, \mathcal{T P P}_{K}^{2}=\left\{T p p_{q}^{1}, \ldots, T p p_{q}^{g}\right\}\right)\right\rangle$ is closed in the data matrix $M$ if, and only if, $\mathcal{C} *$ is closed in the data matrix $M^{\prime}$. Thus, $\mathbb{C}$-closed patterns in $M$ are in one-to-one correspondence with $\mathbb{C P}$-closed patterns in $M^{\prime}$. (Proof is omitted due to page limitation.)

\section{Mining algorithms}

Our efficient mining of all significant pan-correlation patterns consists of the following four components. 


\subsection{Transform time-course data set $M$ into sequential transaction data set $S$}

Given a time-course data set $M=\left[m_{i, j}\right]_{N_{V} \times N_{T}}$, let $s_{i, j}$ be the value movement of the variable $v_{i}$ between time point $t_{j}$ and $t_{j+1}\left(=t_{j}+1\right)$. Specifically, $s_{i, j}$ is $\mathrm{U}$ (up) if $m_{i, j+1} \geq m_{i, j}+\delta_{i}$, and is $\mathrm{D}$ (down) if $m_{i, j+1} \leq m_{i, j}-\delta_{i}$, and is $\mathrm{O}$ otherwise. Let $R_{i}=\left\{s_{i, 1}, s_{i, 2}, \cdots, s_{i, N_{T}-1}\right\}$ be the sequence of all value movements of $v_{i} \in V$. Let $S=\left[s_{i, j}\right]_{N_{V} \times\left(N_{T}-1\right)}$ be a sequential transaction data set which is easily transformed from $M . S$ has the same variables $V$ as $M$ does, but each variable in $S$ has $N_{T}-1$ sequential value movements. In the transformation, $\delta_{i}$ is used to define the scale of the variable $v_{i}$ 's value movement in $M . \delta_{i}$ for $v_{i} \in V$ is set as twenty percents of the absolute difference between the second maximum value of $m_{i, j}$ and the second minimum value of $m_{i, j}, 1 \leq j \leq N_{T}$. The maximum value and the minimum value are discarded to avoid some outlier values of $v_{i}$ in $M$.

We view $S$ as a set of sequential transactions. And each row $R_{i}$ in $S$ corresponds to a sequential transaction and is viewed a sequence of value movements $(\mathrm{U}, \mathrm{D}$, and $\mathrm{O})$. Given any variable $v_{i} \in V$ and any ordered set of time-point pairs Tpp $=\left\{\operatorname{tpp}_{\left(i_{j}, i_{j}+1\right)} \mid j=1,2, \ldots, h\right\}$. Let $f\left(v_{i}, T p p\right)$ be the list $\left\{s_{i, i_{1}}, \ldots, s_{i, i_{h}}\right\}$. Thus, $f\left(v_{i}, T p p\right)$ gives the value movements of $v_{i}$ during $T p p$. We write $f^{\prime}\left(v_{i}, T p p\right)$ to denote the list obtained by flipping every $\mathrm{U}$ to $\mathrm{D}$ and every $\mathrm{D}$ to $\mathrm{U}$ in $f\left(v_{i}, T p p\right)$. In $S$, a sequential pattern is a list of value movements $(\mathrm{U}, \mathrm{D}$, and $\mathrm{O})$. A sequential pattern $s p=\left\{s_{1}, \ldots, s_{h}\right\}$ is said to occur in a sequential transaction $R_{i}$ if there is a list of time-point pairs $T p p=\left\{\operatorname{tpp}_{\left(i_{j}, i_{j}+1\right)} \mid j=1,2, \ldots, h\right\}$, such that $f\left(v_{i}, T p p\right)=s p$. That is, the value movements specified in the pattern $s p$ occur in the transaction $R_{i}$ in the same order as they appear in $s p$, possibly separated by other value movements. We write $\operatorname{supp}(s p, S)$ to denote the support of the sequential pattern $s p$ in $S$.

The space of all sequential patterns occurring in $S$ is denoted by $\mathbb{S P}$. A closed sequential pattern in $\mathbb{S P}$ is defined below, which is similar to those in previous works [13].

Definition 5. Let $s p$ and $s p^{\prime}$ be two sequential patterns. We say $s p \leq s p^{\prime}$ in $\mathbb{S P}$ if, and only if, $s p$ is a subsequence of $s p^{\prime}$ or is identical to $s p^{\prime}$, and $\operatorname{supp}(s p, S)=$ supp $\left(\operatorname{sp}^{\prime}, S\right)$. The closed patterns of $\mathbb{S P}$ are the maximal patterns in $\mathbb{S P}$ according to this partial order.

It is obvious that $f\left(v_{x_{i}}, T p p^{i}\right)=f\left(v_{x_{j}}, T p p^{j}\right)$ for $1 \leq i, j \leq h$, for any pattern $C=\left\langle\mathcal{V}=\left\{v_{x_{1}}, \ldots, v_{x_{h}}\right\}, \mathcal{T} \mathcal{P} \mathcal{P}=\left\{T p p^{1}, \ldots, T p p^{h}\right\}\right\rangle$ in $\mathbb{C P}$ in $M$. The following easily-proved property connects the closed patterns in $\mathbb{S P}$ of $S$ and those in $\mathbb{C P}$ of $M$.

Proposition 2. For every $\mathbb{S P}$-closed pattern $s p$ in $S$, there is a unique $\mathbb{C P}$ closed pattern $\mathcal{C}=\left\langle\mathcal{V}=\left\{v_{x_{1}}, \ldots, v_{x_{h}}\right\}, \mathcal{T P} \mathcal{P}=\left\{T p p^{1}, \ldots, T p p^{h}\right\}\right\rangle$ in $M$, such that $s p=f\left(v_{x_{i}}, T p p^{i}\right)$ for $1 \leq i \leq h$. And for every $\mathbb{C P}$-closed pattern $\mathcal{C}=\langle\mathcal{V}=$ $\left.\left\{v_{x_{1}}, \ldots, v_{x_{h}}\right\}, \mathcal{T} \mathcal{P P}=\left\{T p p^{1}, \ldots, T p p^{h}\right\}\right\rangle$ in $M$, there is a $\mathbb{S P}$-closed pattern sp in $S$, such that $s p=f\left(v_{x_{i}}, T p p^{i}\right)$ for $1 \leq i \leq h$. Thus, $\mathbb{S P}$-closed patterns in $S$ are in one-to-one correspondence with $\mathbb{C P}$-closed patterns of $M$. 


\section{$3.2 \quad$ Opposite mirror copy of $S$}

In $S=\left[s_{i, j}\right]_{N_{V} \times\left(N_{T}-1\right)}$, a positive correlation pattern is denoted by one sequence of value movements, while a negative correlation pattern is denoted by two sequences of value movements whose value movements are opposite to each other at every position, U vs. D, and D vs. U. To make available in $S$ the unified formulation of positive and negative correlation patterns, an opposite mirror copy of each transaction in $S$ is created and added into $S$. This data management technique was similarly used by [8] for mining biclusters.

Given the value movements of $v_{i}$ in $S$, i.e., $R_{i}=\left\{s_{i, 1}, s_{i, 2}, \ldots, s_{i, N_{T}-1}\right\}$, let its opposite mirror copy be $R *_{i}=\left\{s *_{i, 1}, s *_{i, 2}, \ldots, s *_{i, N_{T}-1}\right\}$ where $s *_{i, j}$ is up if $s_{i, j}$ is down, $s *_{i, j}$ is down if $s_{i, j}$ is up, and otherwise $s *_{i, j}=s_{i, j}$. The opposite mirror copy of all transactions in $S$ are added into $S$. The new transaction data set is denoted by $S^{\prime}=\left[s_{i, j}^{\prime}\right]_{2 N_{V} \times\left(N_{T}-1\right)}$, where all $R_{i} \mathrm{~s}$ of $v_{i}$ s are indexed from 0 to $2 N_{V}-2$ with step 2 in $S^{\prime}$, and all $R *_{i}$ s are indexed from 1 to $2 N_{V}-1$ with step 2. This index strategy is used later. $S^{\prime}$ is also the sequential transaction data set derived from $M^{\prime}$.

Then, the crucial theorem below follows immediately from Theorem 1 and Proposition 2.

Theorem 2. SP-closed patterns in $S^{\prime}$ are in one-to-one correspondence with $\mathbb{C}$-closed patterns in $M$. (Proof is omitted due to page limitation.)

\subsection{Mine frequent closed sequential value movements in $S^{\prime}$}

All $\mathbb{S P}$-closed patterns in $S^{\prime}$ can be detected using efficient algorithms of mining closed sequential patterns. After that, given a $\mathbb{S P}$-closed pattern in $S^{\prime}$, by Theorem 2 , there is a corresponding $\mathbb{C P}$-closed pattern in $M^{\prime}$, i.e., a $\mathbb{C}$-closed pattern in $M$. Then, all pan-correlation patterns can be easily obtained from these frequent closed sequential value movements by restoring the time-point pair information and the transaction id information: given a $\mathbb{S P}$-closed pattern $s p$ and its $\operatorname{supp}(s p, S)$ with $\left\{v_{x_{1}}, \ldots, v_{x_{h}}, v_{y_{1}} *, \ldots, v_{y_{g}} *\right\}$, the variables from $V$ of $M^{\prime}$ are grouped in one set while those from $V *$ are grouped in another set, indicating the negative correlation between the two sets; then, the time-point pair information associated with $s p$ is detected by matching $s p$ with each variable $v_{x_{i}} \in \operatorname{supp}(s p, S)$ where there might be multiple matches in $v_{x_{i}}$, indicating multiple occurrence of $s p$ in $v_{x_{i}}$.

\subsection{Opposite mirror copy causes redundancy in patterns}

In $M^{\prime}$, every pan-correlation pattern has a mirror image that carries the same information.For example, a negative correlation pattern $\mathcal{C}=\left\langle\left(\mathcal{V}_{1}, \mathcal{V}_{2}\right),\left(\mathcal{T} \mathcal{P} \mathcal{P}_{1}, \mathcal{T} \mathcal{P} \mathcal{P}_{2}\right)\right\rangle$ in $M$ can be represented by $\mathcal{C} *=\left\langle\mathcal{V}_{1} \cup \mathcal{V}_{2} *, \mathcal{T} \mathcal{P} \mathcal{P}_{1} \cup \mathcal{T} \mathcal{P} \mathcal{P}_{2}\right\rangle$ or $\mathcal{C} *^{\prime}=\left\langle\mathcal{V}_{1} *\right.$ $\left.\cup \mathcal{V}_{2}, \mathcal{T} \mathcal{P} \mathcal{P}_{1} \cup \mathcal{T} \mathcal{P} \mathcal{P}_{2}\right\rangle$ in $M^{\prime}$. Here, $\mathcal{V}_{1} *$ is the negation of $\mathcal{V}_{1}$ and $\mathcal{V}_{2} *$ is the negation of $\mathcal{V}_{2}$. Correspondingly in $S^{\prime}$ from $M^{\prime}, s p=f\left(v_{x_{i}}, T p p^{i}\right)$ for $v_{x_{i}} \in \mathcal{V}_{1} \cup \mathcal{V}_{2} *$ and $s p^{\prime}=f\left(v_{y_{j}}, T p p^{j}\right)$ for $v_{y_{j}} \in \mathcal{V}_{1} * \cup \mathcal{V}_{2}$, and $s p \neq s p^{\prime}$. Thus, $\mathcal{C}$ is mined twice in terms of $s p$ or $s p^{\prime}$ in $S^{\prime}$. And once one of $s p$ and $s p^{\prime}$ is known, there is no need to mine the other because the other can be produced according to the flip relationship between their components. Thus, $s p$ and $s p^{\prime}$ are redundant. It is 
easily proved that a closed $\mathcal{C}$ correlation pattern in $M$ is always detected twice in terms of $s p$ and $s p^{\prime}$ in $S^{\prime}$.

Fortunately, each pair of redundant patterns has some unique property below. Without loss of generality, let a pair of redundant patterns $\mathcal{C} *=\left\langle\left(v_{x_{1}}, T p p_{p}^{1}\right), \ldots,\left(v_{x_{h}}, T p p_{p}^{h}\right)\right.$, $\left.\left(v_{y_{1}} *, T p p_{q}^{1}\right), \ldots,\left(v_{y_{g}} *, T p p_{q}^{g}\right)\right\rangle$ and $\mathcal{C} *^{\prime}=\left\langle\left(v_{x_{1}} *, T p p_{p}^{1}\right), \ldots,\left(v_{x_{h}} *, T p p_{p}^{h}\right),\left(v_{y_{1}}, T p p_{q}^{1}\right), \ldots,\left(v_{y_{g}}, T p p_{q}^{g}\right)\right\rangle$ on $M^{\prime}$ both capture the same information as $\mathcal{C}=\left\langle\left(\mathcal{V}_{1}=\left\{v_{x_{1}}, \ldots, v_{x_{h}}\right\}, \mathcal{V}_{2}=\right.\right.$ $\left.\left.\left\{v_{y_{1}}, \ldots, v_{y_{g}}\right\}\right),\left(\mathcal{T} \mathcal{P} \mathcal{P}_{K}^{1}=\left\{T p p_{p}^{1}, \ldots, T p p_{p}^{h}\right\}, \mathcal{T} \mathcal{P P}_{K}^{2}=\left\{T p p_{q}^{1}, \ldots, T p p_{q}^{g}\right\}\right)\right\rangle$ in $M$. Here $v *$ is the negation of $v$. Then, we rewrite $\mathcal{C} *=\left\{\left(v_{z_{1}}, T p p^{1}\right), \ldots,\left(v_{z_{h+g}}, T p p^{h+g}\right)\right\}$ and $\mathcal{C} *^{\prime}=\left\{\left(v_{w_{1}}^{\prime}, T p p^{1^{\prime}}\right), \ldots,\left(v_{w_{h+g}}^{\prime}, T p p^{h+g^{\prime}}\right)\right\}$. In $\mathcal{C} *$ and $\mathcal{C} *^{\prime}$, assume that all pairs of $\left(v_{z_{*}}, T p p^{*}\right)$ are ordered first according to the transaction indexes of $v_{z_{*}}$ and then according to the time-point pairs in $T p p^{*}$. After that, it is easily proved that $v_{z_{1}}=v_{w_{1}} *$, or $z_{1}=w_{1}$ and $T p p^{1} \leq T p p^{1^{\prime}}$, or vice versa.

Thus to avoid producing redundant $\mathbb{S} \mathbb{P}$-closed patterns in $S^{\prime}$, we must modify the algorithm for mining sequential value movements. We apply two constraints below to prune the redundant patterns. (i) On a sub-dataset $S_{s}^{\prime} \subseteq S^{\prime}$ with the ascending order of the indexes of all transactions on $S^{\prime}$ (Please refer to Section 3.2 for the detail of the indexes), assume $R_{x_{j}}$ is the first transaction on $S_{s}^{\prime}$, i.e., the transaction with the minimum transaction index. If $R_{x_{j}}$ is produced from a $v_{i} * \in V *$, all sequential patterns on $S_{s}^{\prime}$ are redundant and thus the search of new sequential patterns on $S_{s}^{\prime}$ should be pruned. (ii) Otherwise, given a frequent value movement $e$ (i.e. a value movement $\mathrm{U}, \mathrm{D}$ or $\mathrm{O}$ ) on $S_{s}^{\prime}$, let $R_{x_{\min 1}}$ be the transaction with the minimum id where $e$ occurs, and $p o s_{1}$ be the first occurrence

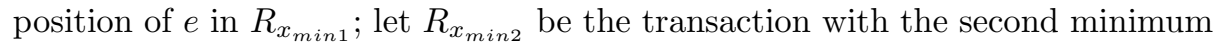
id where $e$ occurs, and $\operatorname{pos}_{2}$ be the first occurrence position of $e$ in $R_{x_{\text {min } 2}}$. If $R_{x_{m i n} 1}$ is produced from $v_{i} \in V$ and $R_{x_{m i n} 2}$ is produced from $v_{i} * \in V *$ and $\operatorname{pos}_{1}>\operatorname{pos}_{2}$, the search in the branch of frequent sequential patterns adding $e$ is redundant and should be pruned. The lemma below is easily proved.

Lemma 2. Our pruning strategy can guarantee that the closed sequential patterns detected are complete and non-redundant in $S^{\prime}$. (Proof is omitted due to page limitation.)

\subsection{Parameter setting}

Three parameters, $\min _{V}, \min _{T P P}$ and $\max _{O}$, are used to prune trivial correlation patterns. In a given pan-correlation pattern $\mathcal{C}=\langle\mathcal{V}, \mathcal{T} \mathcal{P} \mathcal{P}\rangle, \min _{V}$ is the minimum size of $\mathcal{V}, \min _{T P P}$ is the minimum size of $T p p \in \mathcal{T P} \mathcal{P}$, and $\max _{O}$ is the maximum number of $\mathrm{O}$ contained.

\subsection{An illustrative example}

Figure 1 illustrates how our algorithm works. A time-course data set $M$ has six variables and eight time points. $M$ is shown in Figure 1(a) and visualized in Figure 1(b). Figure 1(b) does not easily show a very nice pan-correlation between the six variables. But our algorithm can discover a good negative correlation pattern among the six variables.

By our algorithm, $M$ is firstly discretized to obtain a sequential data $S$ in the first part of Figure 1(c). Then the opposite mirror copy of all sequences in 


\begin{tabular}{|c|c|c|c|c|c|c|c|c|}
\hline ID & 1 & 2 & 3 & 4 & 5 & 6 & 7 & 8 \\
\hline v1 & 5 & 11 & 14 & 8 & 9 & 4 & 13 & $\not$ \\
v2 & 2 & 6 & 9 & $\not \supset$ & $\varnothing$ & 4 & 1 & 8 \\
v3 & 1 & 1 & 3 & 6 & 4 & 0 & 5 & $\not 5$ \\
v4 & -6 & -10 & -14 & -9 & -3 & -13 & $\not 3$ & $\not 3$ \\
v5 & -2 & -5 & -8 & -5 & -1 & -9 & -10 & $\not 5$ \\
v6 & $\not 1$ & $\varnothing$ & 0 & -4 & -7 & -5 & -1 & -5 \\
\hline
\end{tabular}

(a)

\begin{tabular}{|cc|c|c|c|c|c|c|c|}
\hline ID & $\begin{array}{c}2-1 \\
\mathrm{a}\end{array}$ & $\begin{array}{c}3-2 \\
\mathrm{~b}\end{array}$ & $\begin{array}{c}4-3 \\
\mathrm{c}\end{array}$ & $\begin{array}{c}5-4 \\
\mathrm{~d}\end{array}$ & $\begin{array}{c}6-5 \\
\mathrm{e}\end{array}$ & $\begin{array}{c}7-6 \\
\mathrm{f}\end{array}$ & $\begin{array}{c}8-7 \\
\mathrm{~g}\end{array}$ \\
\hline v1 & 0 & $\mathrm{U}$ & $\mathrm{U}$ & $\mathrm{D}$ & $\varnothing$ & $\mathrm{D}$ & $\mathrm{U}$ & $\varnothing$ \\
$\mathrm{v} 2$ & 2 & $\mathrm{U}$ & $\mathrm{U}$ & $\mathrm{D}$ & $\varnothing$ & $\varnothing$ & $\mathrm{D}$ & $\mathrm{U}$ \\
$\mathrm{v} 3$ & 4 & $\varnothing$ & $\mathrm{U}$ & $\mathrm{U}$ & $\mathrm{D}$ & $\mathrm{D}$ & $\mathrm{U}$ & $\varnothing$ \\
v4 & 6 & $\mathrm{D}$ & $\mathrm{D}$ & $\mathrm{U}$ & $\mathrm{U}$ & $\mathrm{D}$ & $\varnothing$ & $\varnothing$ \\
v5 & 8 & $\mathrm{D}$ & $\mathrm{D}$ & $\mathrm{U}$ & $\mathrm{U}$ & $\mathrm{D}$ & $\varnothing$ & $\varnothing$ \\
v6 & 10 & $\varnothing$ & $\varnothing$ & $\mathrm{D}$ & $\mathrm{D}$ & $\mathrm{U}$ & $\mathrm{U}$ & $\mathrm{D}$ \\
\hline \multicolumn{7}{|c|}{ The opposite discritized data set } \\
\hline v1 & 1 & $\mathrm{D}$ & $\mathrm{D}$ & $\mathrm{U}$ & $\varnothing$ & $\mathrm{U}$ & $\mathrm{D}$ & $\mathrm{U}$ \\
v2 & 3 & $\mathrm{D}$ & $\mathrm{D}$ & $\mathrm{U}$ & $\varnothing$ & $\varnothing$ & $\mathrm{U}$ & $\mathrm{D}$ \\
v3 & 5 & $\varnothing$ & $\mathrm{D}$ & $\mathrm{D}$ & $\mathrm{U}$ & $\mathrm{U}$ & $\mathrm{D}$ & $\varnothing$ \\
v4 & 7 & $\mathrm{U}$ & $\mathrm{U}$ & $\mathrm{D}$ & $\mathrm{D}$ & $\mathrm{U}$ & $\varnothing$ & $\varnothing$ \\
v5 & 9 & $\mathrm{U}$ & $\mathrm{U}$ & $\mathrm{D}$ & $\mathrm{D}$ & $\mathrm{U}$ & $\varnothing$ & $\varnothing$ \\
v6 & 11 & $\varnothing$ & $\varnothing$ & $\mathrm{U}$ & $\mathrm{U}$ & $\mathrm{D}$ & $\mathrm{D}$ & $\mathrm{U}$ \\
\hline
\end{tabular}

(c)

\begin{tabular}{|c|c|}
\hline Pattern 1 & $\mathrm{U}, \mathrm{U}, \mathrm{D}, \mathrm{D}, \mathrm{U}$ \\
\cline { 2 - 2 } Occurrence 1 & $0,2,4,7,9,11$ \\
\hline Pattern 2 & $\mathrm{D}, \mathrm{D}, \mathrm{U}, \mathrm{U}, \mathrm{D}$ \\
\cline { 2 - 2 } Occurrence 2 & $1,3,5,6,8,10$ \\
\hline
\end{tabular}

(e)

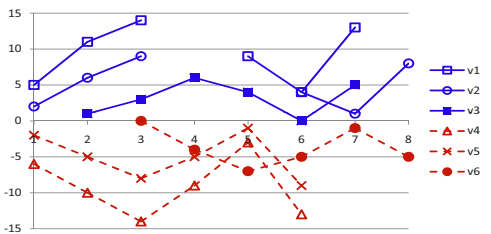

(g)

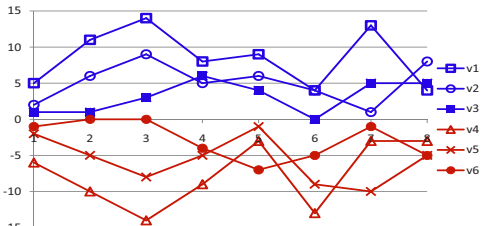

(b)

\begin{tabular}{|cc|c|c|c|c|c|c|c|}
\multicolumn{2}{|c|}{ ID } & $\mathrm{a}$ & $\mathrm{b}$ & $\mathrm{c}$ & $\mathrm{d}$ & $\mathrm{e}$ & $\mathrm{f}$ & $\mathrm{g}$ \\
\hline $\mathrm{v} 1$ & 0 & $\mathrm{U}$ & $\mathrm{U}$ & $\mathrm{D}$ & $\varnothing$ & $\mathrm{D}$ & $\mathrm{U}$ & $\varnothing$ \\
& 1 & $\mathrm{D}$ & $\mathrm{D}$ & $\mathrm{U}$ & $\varnothing$ & $\mathrm{U}$ & $\mathrm{D}$ & $\varnothing$ \\
\hline $\mathrm{v} 2$ & 2 & $\mathrm{U}$ & $\mathrm{U}$ & $\mathrm{D}$ & $\varnothing$ & $\varnothing$ & $\mathrm{D}$ & $\mathrm{U}$ \\
& 3 & $\mathrm{D}$ & $\mathrm{D}$ & $\mathrm{U}$ & $\varnothing$ & $\varnothing$ & $\mathrm{U}$ & $\mathrm{D}$ \\
\hline $\mathrm{v} 3$ & 4 & $\varnothing$ & $\mathrm{U}$ & $\mathrm{U}$ & $\mathrm{D}$ & $\mathrm{D}$ & $\mathrm{U}$ & $\varnothing$ \\
& 5 & $\varnothing$ & $\mathrm{D}$ & $\mathrm{D}$ & $\mathrm{U}$ & $\mathrm{U}$ & $\mathrm{D}$ & $\varnothing$ \\
\hline $\mathrm{v} 4$ & 6 & $\mathrm{D}$ & $\mathrm{D}$ & $\mathrm{U}$ & $\mathrm{U}$ & $\mathrm{D}$ & $\varnothing$ & $\varnothing$ \\
& 7 & $\mathrm{U}$ & $\mathrm{U}$ & $\mathrm{D}$ & $\mathrm{D}$ & $\mathrm{U}$ & $\varnothing$ & $\varnothing$ \\
\hline v5 & 8 & $\mathrm{D}$ & $\mathrm{D}$ & $\mathrm{U}$ & $\mathrm{U}$ & $\mathrm{D}$ & $\varnothing$ & $\varnothing$ \\
& 9 & $\mathrm{U}$ & $\mathrm{U}$ & $\mathrm{D}$ & $\mathrm{D}$ & $\mathrm{U}$ & $\varnothing$ & $\varnothing$ \\
\hline v6 & 10 & $\varnothing$ & $\varnothing$ & $\mathrm{D}$ & $\mathrm{D}$ & $\mathrm{U}$ & $\mathrm{U}$ & $\mathrm{D}$ \\
& 11 & $\varnothing$ & $\varnothing$ & $\mathrm{U}$ & $\mathrm{U}$ & $\mathrm{D}$ & $\mathrm{D}$ & $\mathrm{U}$ \\
\hline
\end{tabular}

(d)

\begin{tabular}{|c|l|c|}
\hline Pattern 1 & \multicolumn{2}{|c|}{ U,U,D,D,U } \\
\cline { 2 - 3 } Occurrence 1 & group 1 & $\mathrm{v} 1, \mathrm{v} 2, \mathrm{v} 3$ \\
& group 2 & $\mathrm{v} 4, \mathrm{v} 5, \mathrm{v} 6$ \\
\hline
\end{tabular}

(f)

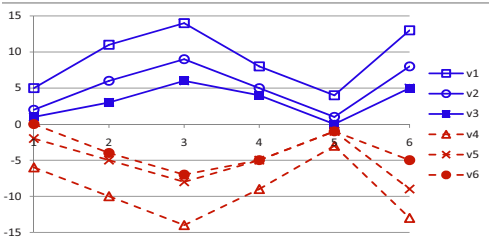

(h)

Fig. 1. An illustrative example of our algorithm. (a) An example of time-course data set $M$. (b) The plot of the example data set. (c) The discretized data set. (d) The combined data set using the opposite mirror copy strategy. (e) The negative pancorrelation patterns. (f) The pattern matching in the original data. (g) The plot of the pattern with gaps and lagged time points. (h) The plot of the pattern merging gaps and ignoring lagged time points (for visualization only). The strike-through numbers, $X, \varnothing$ and $\not \supset$ indicate those values and value movements not in the detected patterns in (e). From (c) to (f), U indicates Up-changed, O no change, while D Down-changed.

$S$, as shown in the second part of Figure 1(c), is constructed using the strategy in Section 3.2. All sequences in Figure 1(c) comprise $S^{\prime}$ in Figure 1(d). With $\min _{V}=5$ and $\min _{T P P}=5$, two pan-correlation patterns are available in $S^{\prime}$, 
as shown in Figure 1(e). It can be clearly seen from Figure 1(e) that these two pan-correlation patterns are the same in the original data $M$, which can be represented in Figure 1(f). Our algorithm can prune the redundancy and only outputs this pattern (visualized in Figure $1(\mathrm{~g})$ ). If the gaps are merged and the time points lagged are ignored (for visualization only), this correlation pattern is shown in Figure 1(h).

\section{Performance evaluation and application}

Our algorithm was tested on both synthetic time-course data sets and real-world time-course data sets of biomedical gene expression. In the implementation, we modified the source code of BIDE+ [13] for detecting pan-correlation patterns by integrating our pruning strategies.

\subsection{Efficiency and scalability results on synthetic data sets}

Two series of synthetic data sets are used. The first series of data sets have the same number of time points but have an increasing number of variables. The second series of data sets have the same number of variables but have an increasing number of time points. The values in these data sets are randomly chosen from $\{-150,-148,-146, \ldots, 150\}$. The efficiency of BIDE+ without our pruning strategies is also evaluated on the mirror-copy datasets of the synthetic data. This performance is used for the comparison to show the contribution of our algorithm.

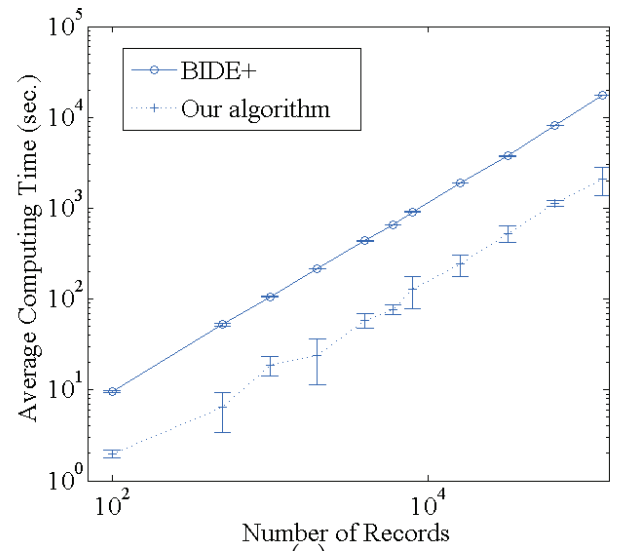

(a)

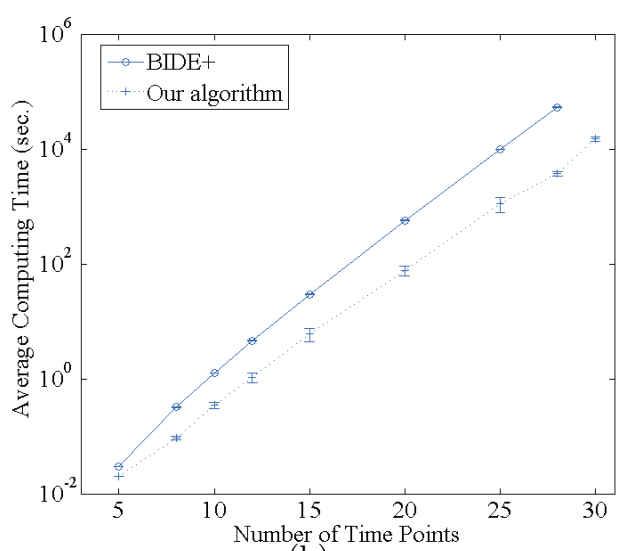

(b)

Fig. 2. The assessment on the synthetic data.Both $\min _{T P P}$ and $\min _{V}$ are set to 2 , and $\max _{O}$ to the number of time points. (a) The computing time (sec.) when the number of variables increases. (b) The computing time (sec.) when the number of time points increases.

Our algorithm was applied to the first series of data sets to see its scalability when the variable size increases. We set the number of time points as $N_{T}=20$, and increase $N_{V}$ from 100 to 500, 1,000, 2,000, 4,000, 6,000, 8,000, 16,000, 32,000, 
64,000 , and to 128,000 . The data at each $N_{V}$ are randomly produced three times to avoid some randomization effect. The average computing time costs are shown in Figure 2(a). It can be seen that the computing time cost by our algorithm increases very slowly. It has approximately linear increment of time complexity with increasing $N_{V}$. In particular when $N_{V}=128,000$, the average computing time is about 30 minutes. Figure 2(a) also shows that BIDE+ without our pruning strategies is more than nine times slower than our algorithm when $N_{V}=128,000$.

Both our algorithm and BIDE+ without our pruning strategies were also applied to the second series of synthetic data sets to examine its scalability when the size of time points increases. We keep the number of variables always as $N_{V}=5000$ and randomly produce data sets with $N_{T}$ varying from 5 to 8 , $10,12,15,20,25,28$, and to 30 . The data sets of each $N_{T}$ are also randomly produced three times to avoid the randomization effect. The average computing time costs are shown in Figure 2(b). The computing costs increase exponentially when the number of time points $N_{T}$ increases. Again, Figure 2(b) suggests that BIDE + without our pruning strategies is more than 14 times slower than our algorithm when $N_{T}=28$. In conclusion, our algorithm is much faster than sequential pattern mining algorithms to detect pan-correlation patterns.

\subsection{Application in time-course gene expression data}

Our algorithm was also evaluated on four real-life microarray gene expression data sets: alpha, cdc15, elu [12], and cdc28 [1]. All of them are time-course gene expression data related to Yeast cell cycle. elu, cdc28, alpha and cdc15 involve 14, 17, 18 and 24 time points, respectively. The four data sets have 5,114 common available genes. our algorithm is able to detect significant pancorrelation patterns efficiently with less than 7 minutes.

At the $\min _{T P P}$ level of $70 \%$ of $N_{T}$ (i.e., spanning at least 10, 12, 13 and 17 time-point pairs in elu, cdc28, alpha and $c d c 15$ respectively), our algorithm detects 1,934 $\mathbb{C}$ pan-correlation patterns in elu, 5,942 in cdc28, 13,693 in alpha and 139,811 in $c d c 15$. Because $\mathbb{C}$ pan-correlation patterns may overlap very much, we filter out overlapping patterns. This filtering results in 588, 2,392, 3,191 and 9,501 non-overlapping $\mathbb{C}$ correlation patterns in elu, cdc28, alpha and cdc15, respectively.

We examine the correlation coefficient, positive or negative, of the variables in our pan-correlation patterns to demonstrate that highly correlated patterns cannot be observed if the time lagging effect or the broken gap is not considered. Given a pan-correlation pattern $\mathcal{C}=\langle\mathcal{V}, \mathcal{T} \mathcal{P} \mathcal{P}\rangle$, its Pearson's correlation coefficient $P C C$ is calculated by $P C C=\frac{\sum_{v_{x_{i}} \in \mathcal{V}, v_{x_{j}} \in \mathcal{V}, x_{i} \neq x_{j}} a b s\left(p\left(v_{x_{i}}, v_{x_{j}}\right)\right)}{(\|\mathcal{V}\| \times(\|\mathcal{V}\|-1))}$, where $a b s(*)$ returns the absolute value of $*, p\left(v_{x_{i}}, v_{x_{j}}\right)$ is the Pearson's correlation coefficient between the value movements of two variables $v_{x_{i}}$ and $v_{x_{j}}$ on all time points in the original time-course data, and $\|\mathcal{V}\|$ is the number of unique variables in $\mathcal{V}$.

In comparison, we also calculate $P C C$ only on $\mathcal{T P} \mathcal{P}$, and call it $P C C^{\mathcal{T P} \mathcal{P}}$. $P C C^{\mathcal{T P P}}$ is also calculated by the above equation except that $p\left(v_{x_{i}}, v_{x_{j}}\right)$ is 
computed only on those time-point pairs involving in $\mathcal{T P \mathcal { P }}$. When $P C C$ or $P C C^{\mathcal{T} \mathcal{P P}}$ is 1 , it means that all the variables in $\mathcal{V}$ are correlated ideally with each other. When $P C C$ or $P C C^{\mathcal{T P P}}$ is 0 , there is completely no correlation for the variables. $P C C$ and $P C C^{\mathcal{T P P}}$ are compared to signify particularly that time-lagged correlation patterns can have strong correlations.

The results are shown in Table 1. It is observed that the variables in our $\mathbb{C}$ pan-correlation patterns are highly correlated with each other, having an average $P C C^{\mathcal{T P P}}>0.82$ across the four datasets. However, their correlation on all timepoint pairs without consideration of time lagging effect or broken gaps is very low with an average $P C C<0.35$ across the four datasets. This implies that if an algorithm does not take lagged time points and gaps into considerations, it would miss many pan-correlation patterns or would discover only specialized pan-correlation patterns.

Table 1. $P C C$ and $P C C^{\mathcal{T P P}}$ on four time-course gene expression data.

\begin{tabular}{|c|c|c|c|c|c|}
\hline Dataset & & $\min ^{a}$ & $\mathrm{mean}^{a}$ & $\mathrm{std}^{a}$ & $\max ^{a}$ \\
\hline elu & $P C C$ & 0.191 & 0.294 & 0.026 & 0.450 \\
\hline & $P C C^{\text {TPPP }}$ & 0.719 & 0.832 & 0.022 & 0.923 \\
\hline cdc28 & $P C C$ & 0.069 & 0.264 & 0.036 & 0.483 \\
\hline & $P C C^{\text {TPPP }}$ & 0.657 & 0.827 & 0.028 & 0.919 \\
\hline alpha & $P C C$ & 0.133 & 0.299 & 0.048 & 0.565 \\
\hline & $P C C^{\mathcal{T P P}}$ & 0.685 & 0.832 & 0.029 & 0.936 \\
\hline cdc15 & $P C C$ & 0.122 & 0.347 & 0.083 & 0.799 \\
\hline & $P C C^{\text {TPP }}$ & 0.620 & 0.826 & 0.034 & 0.933 \\
\hline
\end{tabular}

${ }^{a}$ : The minimum, mean, standard deviation and maximum $P C C$ or $P C C^{\mathcal{T P \mathcal { P }}}$ of all pan-correlation patterns in each data set.

Four examples of pan-correlation patterns We show one pan-correlation pattern for each of the four microarray time-course data sets to partly illustrate the complexity of mining correlation patterns. These examples are displayed at Figure $3(\mathrm{c}), 3(\mathrm{~d}), 3(\mathrm{~g})$ and $3(\mathrm{~h})$. The original time-course data of the involved variables are also presented in Figure 3. From Figure 3(a), 3(b), 3(e) and 3(f), we can see that pan-correlation patterns are hardly visualized in the background of original data due to the gaps and lagged time points. However, these pancorrelation patterns turn out to be clear, as shown in Figure 3(c), 3(d), 3(g) and $3(\mathrm{~h})$, after the removal of gaps and shifting. There are many similar examples we found from the four Yeast cell time-course gene expression data sets. Their biological significance is strong (the result is not reported here as that is a different topic).

\section{Conclusion}

In this work, we have proposed an efficient algorithm for mining all significant pan-correlation patterns from time-course data sets based on three effective ideas: the discretization idea, the generalized representation of positive patterns 


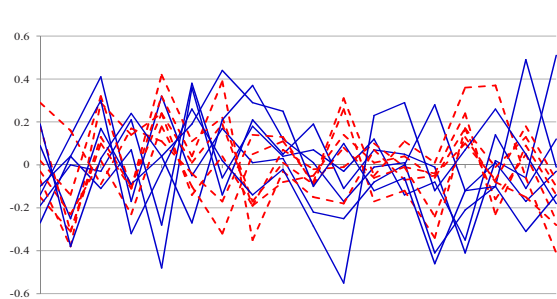

(a) $\stackrel{3}{P}^{3} C C^{5}=0.378^{9}$ on all time points

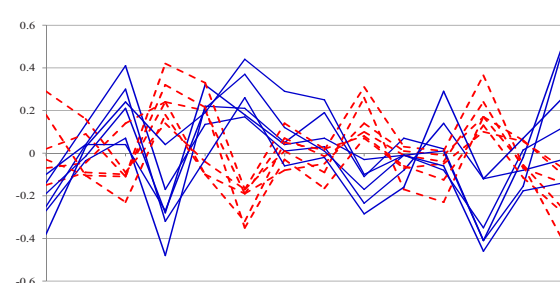

(c) A pan-correlation pattern "in alpha with $P C C^{\mathcal{T P P}}=0.844$

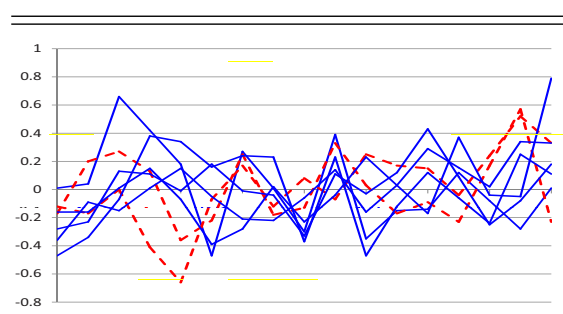

(e) $P^{3} C C \stackrel{5}{=} 0.3^{7} 19 \stackrel{9}{\text { on }}$ all time points ${ }^{13}$

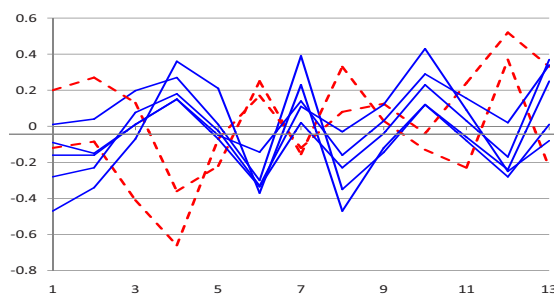

(g) A pan-correlation pattern in ${ }^{7} d c 2^{13}$ with $P C C^{\mathcal{T P \mathcal { P }}}=0.851$

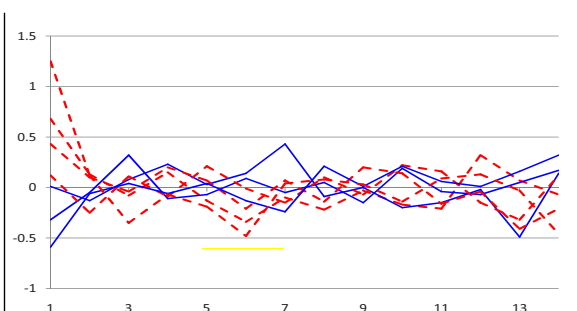

(b) $P C^{3} C=\stackrel{5}{5}^{1} .346^{7}$ on all time points

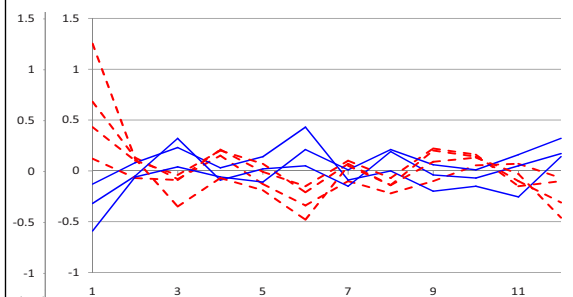

(d) A A $^{3}$-correlation ${ }^{7}$ pattern in ${ }^{11}$ elu with $P C C^{\mathcal{T P P}}=0.809$

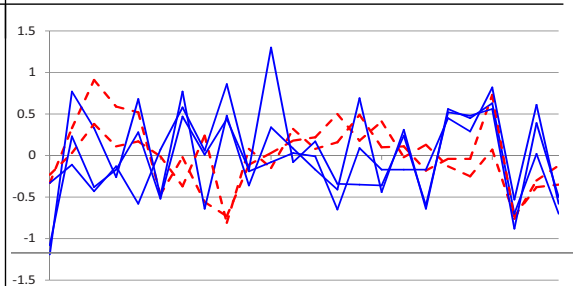

(f) $P C C^{6}=0.343^{11}$ on all time points

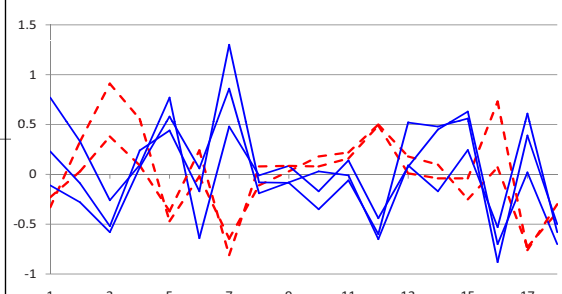

(h) $)^{1} \stackrel{3}{~ p a n-c o r r e l a t i o n ~}^{-11}{ }^{13}$ pattern $^{15}$ in $^{17} c d^{15}$ with $P C C^{\mathcal{T P \mathcal { P }}}=0.870$

Fig. 3. Four examples of pan-correlation patterns with two sets of variables: one set with solid blue line and the other with dashed red line. (a), (b), (e) and (f): The original time-course data of the involved variables in the four pan-correlation pattern examples on alpha, elu, cdc28 and cdc15 data set of Yeast cell cycle, respectively. (c), (d), (g) and $(\mathrm{h})$ : The corresponding pan-correlation pattern with smoothing after removing time-lagged points and gaps. Small errors may be in the pattern due to smoothing.

and the opposite-mirror copy of the original sequential data set. Our algorithm 
has been tested on synthetic time-course data sets and on four Yeast cell cycle time-course data sets. The efficiency of our algorithm has shown to be high.

\section{References}

1. Cho, R.J., Campbell, M.J., Winzeler, E.A., Steinmetz, L., Conway, A., Wodicka, L., Wolfsberg, T.G., Gabrielian, A.E., Landsman, D., Lockhart, D.J., Davis, R.W.: A genome-wide transcriptional analysis of the mitotic cell cycle. Molecular cell 2(1), 65-73 (1998)

2. Chuang, C.L., Jen, C.H., Chen, C.M., Shieh, G.S.: A pattern recognition approach to infer time-lagged genetic interactions. Bioinformatics 24(9), 1183-1190 (2008)

3. Getz, G., Levine, E., Domany, E.: Coupled two-way clustering analysis of gene microarray data. Proceedings of the National Academy of Sciences 97(22), 12079$12084(2000)$

4. Ji, L., Tan, K.L.: Mining gene expression data for positive and negative co-regulated gene clusters. Bioinformatics 20(16), 2711-2718 (2004)

5. Ji, L., Tan, K.L.: Identifying time-lagged gene clusters using gene expression data. Bioinformatics 21(4), 509-516 (2005)

6. Jiang, D., Pei, J., Ramanathan, M., Tang, C., Zhang, A.: Mining coherent gene clusters from gene-sample-time microarray data. In: Proceedings of the tenth ACM SIGKDD international conference on Knowledge discovery and data mining. pp. 430-439. KDD '04, ACM, New York, NY, USA (2004)

7. Li, J., Liu, Q., Zeng, T.: Negative correlations in collaboration: concepts and algorithms. In: KDD. pp. 463-472 (2010)

8. Madeira, S., Oliveira, A.: A polynomial time biclustering algorithm for finding approximate expression patterns in gene expression time series. Algorithms for Molecular Biology 4(1), 8 (2009)

9. Madeira, S.C., Teixeira, M.C., Sa-Correia, I., Oliveira, A.L.: Identification of regulatory modules in time series gene expression data using a linear time biclustering algorithm. IEEE/ACM Transactions on Computational Biology and Bioinformatics 7(1), 153-165 (2010)

10. Prelic, A., Bleuler, S., Zimmermann, P., Wille, A., Bhlmann, P., Gruissem, W., Hennig, L., Thiele, L., Zitzler, E.: A systematic comparison and evaluation of biclustering methods for gene expression data. Bioinformatics 22(9), 1122-1129 (2006)

11. Roy, S., Bhattacharyya, D.K., Kalita, J.K.: CoBi: Pattern based co-regulated biclustering of gene expression data. Pattern Recognition Letters 34(14), 1669-1678 (2013)

12. Spellman, P.T., Sherlock, G., Zhang, M.Q., Iyer, V.R., Anders, K., Eisen, M.B., Brown, P.O., Botstein, D., Futcher, B.: Comprehensive identification of cell cycleCregulated genes of the yeast saccharomyces cerevisiae by microarray hybridization. Molecular Biology of the Cell 9(12), 3273-3297 (1998)

13. Wang, J., Han, J.: BIDE: efficient mining of frequent closed sequences. In: Data Engineering, 2004. Proceedings. 20th International Conference on. pp. 79-90 (2004)

14. Zeng, T., Li, J.: Maximization of negative correlations in time-course gene expression data for enhancing understanding of molecular pathways. Nucleic Acids Research 38(1), e1 (2010)

15. Zhao, Y., Yu, J., Wang, G., Chen, L., Wang, B., Yu, G.: Maximal coregulated gene clustering. Knowledge and Data Engineering, IEEE Transactions on 20(1), 83-98 (2008) 